\title{
If You Can't Be With the One You Love, Love the One You're With: How Individual Habituation of Agent Interactions Improves Global Utility
}

\author{
Adam P. Davies* ${ }^{1}$, Richard A. Watson ${ }^{1}$, Rob Mills ${ }^{1}$, C. L. Buckley ${ }^{2}$, Jason Noble ${ }^{1}$ \\ ${ }^{1}$ Natural Systems group, ECS, University of Southampton, U.K. \\ ${ }^{2}$ CCNR, University of Sussex, U.K. \\ Email: apd1e09@ecs.soton.ac.uk (A.P.D); raw@ecs.soton.ac.uk (R.A.W); rob.mills@soton.ac.uk (R.M.); c.1.buckley@sussex.ac.uk \\ (C.L.B); jn2@ecs.soton.ac.uk (J.N.) \\ *Corresponding authour
}

Keywords: Hebbian Learning, Hopfield Network, associative memory, game theory, self-organisation, adaptive networks

\begin{abstract}
Simple distributed strategies that modify the behaviour of selfish individuals in a manner that enhances cooperation or global efficiency have proved difficult to identify. We consider a network of selfish agents who each optimise their individual utilities by coordinating (or anti-coordinating) with their neighbours, to maximise the pay-offs from randomly weighted pair-wise games. In general, agents will opt for the behaviour that is the best compromise (for them) of the many conflicting constraints created by their neighbours, but the attractors of the system as a whole will not maximise total utility. We then consider agents that act as 'creatures of habit' by increasing their preference to coordinate (anti-coordinate) with whichever neighbours they are coordinated (anti-coordinated) with at the present moment. These preferences change slowly while the system is repeatedly perturbed such that it settles to many different local attractors. We find that under these conditions, with each perturbation there is a progressively higher chance of the system settling to a configuration with high total utility. Eventually, only one attractor remains, and that attractor is very likely to maximise (or almost maximise) global utility. This counterintuitive result can be understood using theory from computational neuroscience; we show that this simple form of habituation is equivalent to Hebbian learning, and the improved optimisation of global utility that is observed results from well-known generalisation capabilities of associative memory acting at
\end{abstract}


the network scale. This causes the system of selfish agents, each acting individually but habitually, to collectively identify configurations that maximise total utility.

\section{Selfish Agents and Total Utility}

This paper investigates the effect of a simple distributed strategy for increasing total utility in systems of selfishly optimising individuals. In closely related work we have developed a general model addressing this topic [1], and here we focus on a social agent system and the implications for social networks. The broader topic concerns many different types of systems.

For example, in technological systems, it is often convenient or necessary to devolve control to numerous autonomous components or agents that each, in a fairly simple manner, acts to optimise a global performance criterion: e.g. communications routing agents act to minimise calls dropped, or processing nodes in a grid computing system each act to maximise the number of jobs processed $[2,3]$. However, since each component in the network acts individually, i.e., using only local information, constraints between individuals can remain unsatisfied, resulting in poorly optimised global performance. In an engineered system one could, in principle, mandate that all nodes act in accord with the globally optimal configuration of behaviours (assuming one knew what that was) - but this would defeat the scalability and robustness aims of complex adaptive systems. The question for engineered complex adaptive systems then, is the question of how to cause simple autonomous agents to act 'smarter' in a fully distributed manner such that they better satisfy constraints between agents and thereby better optimise global performance.

Meanwhile, in evolutionary biology it appears that in certain circumstances symbiotic species have formed collaborations that are adaptive at a higher level of organisation [4], but it has been difficult to integrate this perspective with the assumption that under natural selection such collaborations must be driven by the selfish interests of the organisms involved [5,6]. 
In social network studies there is increasing interest in adaptive networks [7] where agents in a network can alter the structure of the connections in the network. Of particular interest is the possibility that by doing so they may increase the ability of the system to maintain high levels of cooperation $[8,9,10,11]$. However, despite increased focus on models of 'network reciprocity' [12], a general understanding of how agents on a network modify their interactions with others in a way that increases total cooperation is poorly understood.

In each domain we are, at the broadest level, interested in understanding/identifying very simple mechanisms that might cause self-interested agents to modify their behaviour, or how their behaviours are affected by others, in a manner that increases adaptation or efficiency either globally or at a higher-level of organisation than the individual.

Taking an agent perspective, the obvious problem is this: If it is the case that agents collectively create adaptation that is not explained by the default selfish behaviours of individuals, then it must be the case that, on at least some occasions, agents make decisions that are detrimental to individual interests. If this were not the case then there is nothing to be explained over and above the selfish actions of individuals. But if it is the case, then this appears to run counter to any reasonable definition of a rational selfish agent. In what sense could it be self-consistent to suggest that a selfish agent has adopted a behaviour that decreased individual utility? One way to make sense of this is the possibility that, at the time that the agent takes this action, it appears to them be the best thing for them - that the agent is no longer making decisions according to the true utility function but some distortion of it that alters their perception of the utility of that action. If somehow the perception of an agent were distorted in the right way, so that the action that it preferred, the one that it thought was best for it, was in fact the action that was globally optimal, then a rational agent with this distorted set of preferences could increase global efficiency even at the cost of personal utility. 
One might assume that this is easier said than done - but in this paper we suggest that the reverse is true; it is easier to do than to explain how it works. However, the general problem and the essence of the strategy we investigate is straightforwardly introduced by means of the following simple parable.

Although this makes the concepts intuitively accessible it might tend to cast the model in a narrow interpretation - it is, of course, not really a model about scientists and their drinking habits, but a general model of interacting agents on a network with pair-wise constraints between binary behaviours.

Consider a community of individuals (e.g. researchers) in a social network. Each has an intrinsic symmetric compatibility, or 'complementarity', with every other individual that determines the productivity/pay-off of collaborating with them. Each evening all researchers attend one of two intrinsically equal public houses (or other such collaborative projects) initially at random. Individuals must decide which to attend based solely on who else attends that venue. Each individual seeks to maximise their scientific productivity by attending the pub that, on that night, maximises the sum of compatibilities with other researchers and minimises incompatibilities. Assessing the company they find at any moment, individuals therefore (one at a time in random order) may choose to switch pubs to maximise their productivity according to the locations of others. Since each individual has compatibilities and incompatibilities with all other individuals, each must choose the pub that offers the best compromise of these conflicting interests. Since compatibilities are symmetric, the researchers will quickly reach a configuration where no one wants to change pubs [13], however, this configuration will not in general be the arrangement that is maximal in total productivity, but merely a locally optimal configuration.

This describes the basic behaviour of agents on the network. Our aim is to devise a simple individual strategy that causes researchers to make better decisions about when to change pubs such that total productivity is maximised. This will necessarily mean that some researchers, at some moments in time, must change pubs even though it decreases their individual productivity. 
Surprisingly, we find that this can be achieved (over many evenings) by implementing a very simple rule each individual must develop a preference for drinking with whichever other researchers they are drinking with right now. As Crosby, Stills and Nash put it “If you can't be with the one you love, honey, love the one you're with" [14]. Since we already know the arrangements of researchers will be initially random and, most of the time, at best sub-optimal, this seems like a counter productive strategy. But, in fact we find that it is capable, given enough evenings and slowly developed preferences, of causing all researchers to develop preferences that cause them to make decisions that maximise total productivity reliably every evening.

The agents that we model are therefore not selfish in an identical way to default agents - they sometimes take actions that do not maximise individual utility, which is the point of the exercise after all. But neither are they overtly cooperative or altruistic agents. They are simply habitual selfish agents. In this paper we are not directly addressing why it might be that selfish agents act as creatures of habit, although we will discuss this briefly. But we suggest this type of distorted perception of a true utility function, one which agents come to prefer familiarity over otherwise obvious opportunities for personal gain, is one which does not require any teleological or, certainly, any centralised control and is therefore relevant to many domains. This 'distorted perception' model differs from the work described in [1], where agents are assumed to be able to directly alter their real constraints with other agents. Watson et al [1] also discuss the immediate selfish benefits that might motivate changes to constraints given that assumption, whereas in this paper we simply mandate that agents act in a habitual manner (see Discussion). But both papers have in common the idea of a self-modelling dynamical system and its equivalence with neural network models [15].

In the next two sections we will detail an illustration of this strategy and the results we observe. In the Discussion section we will outline how this result can be interpreted in terms of adaptive network restructuring. Briefly: Initially, interactions between agents are governed by a network of intrinsic 
constraints (compatibilities), and latterly they are governed by a combination of these intrinsic constraints plus the interaction preferences that the agents have developed. The new behavioural dynamics of the agents caused by interaction preferences can therefore equally be understood as a result of changes to connection strengths in the effective interaction network. The increased global utility observed can then be explained using theory from computational neuroscience. In particular, we can understand how the system as a whole improves global adaptation via the observation that when each agent acts as a creature of habit it changes the effective dependencies in the network in a Hebbian manner $[16,17]$. This means that through the simple distributed actions of each individual agent, the network as a whole behaves in a manner that is functionally equivalent to a simple form of learning neural network [15]. In this case, the network is not being trained by an external training set, but instead is 'learning' its own attractor states, as we will explain. We discuss how a separation of the timescales for behaviours on the network and behaviours of the network (i.e. changes to network structure) is essential for this result, and consider conditions under which a habitual policy could spontaneously arise. We examine the concept of selfishness when applied to longterm utility maximisation mechanisms, and put forward an interpretation of selfishness that reframes the 'conundrum of cooperation', removing the apparent paradox by viewing cooperation as a by-product of long term selfishness. Finally, we link the habituation policy with existing reinforcement -learning algorithms - in particular, those of generalised weakened fictitious play [18].

\section{Methods}

\section{$2.1 \quad$ Default agents}

Our model involves $N=100$ agents playing two-player games (Table 1) on a fully connected network. Specifically, for each game (i.e. each connection in the network), there is a single symmetric payoff matrix, $U_{i j}$, which defines for agents $i$ and $j$ either a coordination game $(\alpha=1, \beta=0)$ or anti-coordination game $(\alpha=0$, $\beta=1$ ) with equal probability (Table 1 ). 
Initially all agents in the network are assigned a behaviour at random, and then the game progresses in extensive form with perfect monitoring, where each agent, in a random order, is permitted to update its behaviour (to either A or B) after observing the current actions of the other players. Each agent does so according to a best response strategy, i.e., to adopt the behaviour (choose a pub) that maximises its utility, $u_{i}$ (Eq.1) given the behaviours (pub choices) currently adopted by its neighbours:

$u_{i}(t)=\sum_{j}^{N} U_{i j}\left(s_{i}(t), s_{j}(t)\right)$

where $U_{i j}(x, y)$ is the payoff received by player $i$ when player $i$ plays strategy $x$ and player $j$ plays strategy $y$ (according to Table 1 above), and $s_{n}(t)$ is the strategy currently played by agent $n$. Behaviours are updated in this manner repeatedly. Each agent is involved in games with all neighbours simultaneously but can adopt only one behaviour at any one time, thus coordinating with one neighbour may preclude coordinating with another, and so each agent must therefore adopt the behaviour that is the best compromise of these constraints. By using a symmetric game, $U_{i j}=U_{j i}$, we can ensure that the system will reach a stable fixed point [13], i.e. a configuration where no agent wants to change behaviour unilaterally. Moreover, this configuration will be a local optimum in the total or global utility, $G$, of the system which is simply the sum of individual utilities [13] (Eq.2).

$$
G(t)=\sum_{i}^{N} \sum_{j}^{N} U_{i j}\left(s_{i}(t), s_{j}(t)\right)
$$

Equivalently, since each game is a potential game [19] and the global game is simply an addition of all local games, then the global game must also be a potential game [20] (the potential function given by Equation 2), and hence will have only pure Nash equilibria [19]. However, in general, the stable configuration reached from an arbitrary initial condition will not be globally maximal in total utility. If the system is repeatedly perturbed (reassigning random behaviours to all agents) at infrequent intervals (here every 1000 time steps = one evening), and thereby allowed to settle, or relax, to many different local equilibria 
(on different evenings), the behaviour of the system given these default agents can be described by the distribution of total utilities found at the end of each of these 'relaxations' (Fig. 1.c).

\section{$2.2 \quad$ Creatures of habit}

We seek a simple distributed strategy that causes agents to make different (hence unselfish) behavioural choices in particular contexts in such a manner that configurations of higher global utility are attained or high global utility configurations are attained with greater reliability (i.e. from a greater number of random initial conditions). To this end we investigate agents that act as 'creatures of habit' by increasing their preference to coordinate with whichever neighbours they are coordinated with at the present moment (regardless of whether this is presently contributing positively or negatively to their utility). Specifically, in addition to the 'true' utility matrix, $U_{i j}$, each agent also possesses a 'preference' matrix, $P_{i j}$, for each of its connections. These are used to modify the behaviour of the agent such that it chooses the behaviour that maximises its 'perceived utility', $p_{i}$, (Eq.3), instead of its true utility (Eq.2) alone:

$p_{i}(t)=\sum_{j}^{N}\left[U_{i j}\left(s_{i}(t), s_{j}(t)\right)+P_{i j}(t)\left(s_{i}(t), s_{j}(t)\right)\right]$

where $P_{i j}$ is a pay-off matrix that represents an agent's preference for the combination of behaviours $s_{i}$ and $s_{j}$. The perceived utility is thus simply the sum of the true utility plus the agent's preferences. Each agent has a separate preference pay-off matrix for each other agent. All preference payoff matrices are initially set to zero, such that the initial dynamics of the agents are as per the default agents. But as the values in these matrices change over time they may come to collectively overpower the tendency to maximise true utility and thereby cause agents to make different decisions about which behaviour is best for them to adopt.

It should be clear that it is possible in principle, with knowledge of the globally optimal system configuration, to assign values to each of the $P_{i j}$ matrices that will cause agents to adopt behaviours that 
maximise global system utility instead of choosing behaviours that maximise individual utility and thereby failing to maximise total utility. But our question then becomes how to enable agents to develop, via a simple distributed strategy (without knowledge of the global optimum, of course) such a perception of interactions with others that causes them to make these globally optimal decisions.

The strategy we investigate is very simple - we assert that each $P_{i j}$ matrix is updated so as to increase the agent's perceived utility at the current moment. Specifically, whenever an agent's behaviour has just been updated (whether it changed behaviour or not), with probability $r_{p}=0.0001$ all of its $P_{i j}$ matrices will also be updated. To decide how to update each $P_{i j}$ matrix, one of two possibilities is considered (chosen at random), either $P_{i j}^{\prime}=P_{i j}(t)+A$ or $P_{i j}^{\prime}=P_{i j}(t)-A$, where $A$ is the adjustment matrix defined in Table 2 . If $p_{i}(t)_{\text {_given }} P_{i j}^{\prime}>p_{i}(t)_{-}$given_ $P_{i j}$ then $P_{i j}(t+1)=P_{i j}^{\prime}$ else $P_{i j}(t+1)=P_{i j}$.

This strategy has the effect of increasing agent $i$ 's preference for coordinating or anti-coordinating with agent $j$ according to whether it is currently coordinating or anti-coordinating with agent $j$, respectively. Note that this preference is not sensitive to whether the interaction between these two agents is currently contributing positively to the utility of agent $i$; an agent increases its preference for the current combination of behaviours irrespective of whether $U_{i j}\left(s_{i}(t), s_{j}(t)\right)>0$. It is thereby simply reinforcing a preference for doing more of what it is currently doing with respect to coordinating with others (i.e. I'm in the same pub with them now, therefore I change my preference so that I like being in the same pub with them a little more or, at least, dislike it less); hence the reference to the Crosby, Stills and Nash song in our title. This is a counterintuitive strategy in the sense that it can increase the preference for coordinating with other agents even when $U_{i j}$ defines an anti-coordination game, and vice versa. Note that this habituation does not alter the independent preference for playing behaviour A or B, but instead alters the preference for coordinating behaviours with others.

\section{Results}


The system is run for 1000 relaxations, of 1000 time steps each, without habituation (i.e. default agents). Example trajectories of total utility for individual relaxations are shown in Fig1.a. The total utility at the end point of each relaxation is shown in Fig.1.b (first 1000 relaxations). The system is then run for 1000 relaxations with habituation (i.e. $r=0.005$ ). As the preference utility matrices change over time the distribution of local optima found changes (Fig.1.b, relaxations 1001-2000). We see in these figures that the probability of finding the configurations with high total utility increases over time. After this habituation period the system is then tested with 1000 further relaxations (using the habituated agents but with no further habituation). The plotted results show that the trajectories of the system after habituation (Fig.1.c) find high-utility configurations reliably. Histograms of the total utilities found before and after habituation are shown in Fig.1.d.

These results therefore show that habituation of agent interactions, created by developing a preference for whatever combination of behaviours is currently observed, has the effect of causing agents to adopt different behaviours in some situations (essentially because the resulting combination of behaviours has been experienced more often in the past). Specifically, since without habituation agents adopt behaviours that maximise their individual (true) utility, so the different behaviours adopted with habituation are therefore behaviours that (at least temporarily) decrease their true utility - otherwise the trajectories would not be different (neutral changes are very rare in this system). Over time agents therefore come to choose behaviours that decrease their individual utility in certain circumstances, but that allow the system to ultimately reach states of global utility higher than would have been otherwise possible. Accordingly, trajectories before and after habitation are different, but more specifically, the behavioural choices that agents make after habituation increase total system utility and are in this well-defined sense more cooperative.

Results collected for 50 independent simulations (each consisting of 1000 relaxations before habitation, 1000 relaxations during habituation and 1000 relaxations after habituation) show that with these 
parameters, the global utility of system configurations found after habituation is on average in the 93rd percentile of global utilities of system configurations found before habituation. This represents a considerable increase in the likelihood of finding a high utility system configuration, but the current learning rate $(\mathrm{r}=0.005)$ does not always cause the system to ultimately settle exactly on the global optimum. In general, as one might expect, there is a trade-off between the speed with which learning can improve global adaptation and the probability that the system will ultimately settle on the globally maximal attractor. But, a sufficiently low learning rate will find the global optimum utility configuration with high probability $[15,21]$.

\section{Discussion}

\subsection{Adaptive networks}

An agent system where actions are governed by a perceived utility (rather than the true utility) is formally equivalent to a system where actions are governed by a new network of constraints (rather than the original network of constraints) [22]. Here we have been modelling a system that is fully connected with coordination and anti-coordination games played on the edges of that network. This is equivalent to a weighted network, where edges are weighted by $\omega_{i j}= \pm 1$, and all games are coordination games $(\alpha=1, \beta=0)$ with pay-off $\omega_{i j} U_{i j}$. (i.e. each of the table entries in $U_{i j}$ is multiplied by the scalar $\omega_{i j}$ ). The structure of the games defined by the pay-off matrices is thus converted into the weighted connections of the network (with identical pay-off matrices). Further, the addition of a preference matrix (restricted to the limited form investigated here) is equivalent to an alteration of this weighting; specifically, $\left(\omega_{i j}+k_{i j} r\right) U_{i j}$, where $r$ is the learning rate (as above) and $k_{i j}$ is the number of times agents $i$ and $j$ have been coordinated in the past minus the number of times they have been anti-coordinated (note that $k_{i j}$ will always equal $k_{j i}$, ensuring that the connections remain symmetric if they start symmetric). Thus, although conceptually contrasting, changing the perception of pay-offs for agent $i$ via a preference matrix is functionally identical to altering the connection strengths between the agents. We chose not to introduce the model in these terms, in part 
because it is important to realise that although an agents' behaviours will be governed by the new connections, the effects on global 'true' utility that we are interested in must be measured using the original connection strengths [15] (it should be clear that if this were not the case it would be trivial for agents to alter connections in a manner that would make satisfying constraints easier for them and thereby increase total utility).

Nonetheless, this perspective helps us to connect the current work with studies of adaptive networks $[23,24,7]$ where agents on a network can alter the topology (here, connection strengths) of connections in the network. We can thereby understand the system we have illustrated to be an example of how agents on a network can 're-structure' the network in a manner that enhances the resolution of conflicting constraints and thereby global efficiency. Other works in this area include that of $[8,9]$ where agents on a network, playing a variety of games, re-wire their links when their utility is low, but keep the local topology unchanged if their utility is high. Although there are several important technical differences with the current work, the basic intuition that agents should alter network topology to make themselves happier (or at least, alter it if they are unhappy) is common to both [1].

In essence, the form of habituation we model is a very simple form of re-structuring; it simply asserts that connections between agents increase or decrease in strength in a manner that reinforces the current combinations of behaviours observed. The effects of this habituation are put into context by considering the problem at hand: we are dealing with a limited form of global optimisation problem [25] in which local optima (and the global optimum) are created by the inability to resolve many overlapping low-order dependencies $[26,15]$. When using simple local search on this problem (i.e. agents without habituation), there is only a small probability of finding configurations with high global utility (Fig.1.a and b); however, they are found nonetheless. Habituation outcompetes local search, not by finding new configurations of absolute higher utility (although this may occur in some cases), but instead by progressively increasing the probability of finding high utility configurations, until only one configuration is ever found (which is very 
likely to be one of high utility). We can therefore view habituation as a mechanism that gradually transforms the search space of the problem from one with many varied local optima, to one with a single (and very likely high utility) optimum, which will always be reached; furthermore, it does so via a simple distributed strategy $[1,15]$.

Specifically, although it is not immediately obvious from a static analysis of the connection matrix which connections should be increased and which decreased in order to cause selfish agents to solve the problem better, the necessary information is naturally revealed by allowing the system to repeatedly settle to local optima and reinforcing the correlations in behaviours so created. These correlations are determined by the connections of the original network in an indirect manner. For example, a particular constraint may often remain unsatisfied in locally optimal configurations even though the direct connection defining this constraint states that it is just as valuable to satisfy it as any other connection. Then if a constraint is often easily satisfied its importance is strengthened, if it is equally often satisfied and unsatisfied it remains unchanged on average, and when agents are on average unable to satisfy it its importance is weakened and eventually its sign can be reversed. This causes the system to, gradually over time, pay more attention to the connections that can be simultaneously satisfied and weaken or soften the constraints that cannot be satisfied. One way to understand the result of this adaptive constraint relaxation/exaggeration is that agents become complementary specialists, i.e. selectively attuned to some constraints more than others. That is, whereas the default agents are generalists who persist in trying to satisfy all constraints whether satisfiable or unsatisfiable, habituating agents, through the self-organisation of the behaviours on the network, come to specialise in a manner that 'for their own comfort' (i.e. for the immediate increase of their perceived utility) fits together better with one another but thereby actually resolves more of the system constraints in total.

\subsection{Self-structuring adaptive networks, neural network learning and associative memory}


How this type of adaptive network, with very simple, local modification of connections, comes to maximise global utility can be explained formally using theory from computational neuroscience. Specifically, the behaviour of the network of default agents detailed above is identical to the behaviour of the discrete Hopfield network [13] (which is just a bit-flip hill-climber [20]) and when connections between nodes increase or decrease in strength in a manner that reinforces the current combinations of behaviours this is formally equivalent to Hebbian learning [1]. Hebb's rule, in the context of neural network learning, is often represented by the slogan neurons that fire together wire together, meaning that synaptic connections between neurons that have correlated activation are strengthened. This learning rule has the effect of transforming correlated neural activations into causally linked neural activations, which from a dynamical systems perspective, has the effect of enlarging the basin of attraction for the current activation pattern/system configuration. This type of learning can be used to train a recurrent neural network to store a given set of training patterns [13] thus forming what is known as an 'associative memory' of these patterns. A network trained with an associative memory then has the ability to 'recall' the training pattern that is most similar to a partially specified or corrupted test pattern.

Formally, a common simplified form of Hebb's rule states that the change in a synaptic connection strength $\omega_{i j}$ is $\Delta \omega_{i j}=\delta s_{i} s_{j}$ where $\delta>0$ is a fixed parameter controlling the learning rate and $s_{n}$ is the current activation of the $n^{\text {th }}$ neuron. Here by changing the pay-off matrix of each individual by $k_{i j}(t) r U_{i j}$ where $k_{i j}(t)$ is the correlation of behaviours at time $t$, we are effecting exactly the same changes. Thus the habituating agents each modify their perceived utilities in a manner that effects Hebbian changes to connection strengths which they must if these preferences are to mean that this behaviour combination is preferred more. This equivalence at the agent level has the consequence that the system of agents as a whole implements an associative memory. Since this is a self-organised network, not a network trained by some external experimenter, this is not an associative memory of any externally imposed training patterns. Rather this is an associative memory of the configuration patterns that are commonly experienced under the networks intrinsic dynamics - and given the perturbation and relaxation protocol we have adopted, which means 
that the system spends most of its time at locally optimal configurations, it is these configurations that the associative memory stores (or enlarges).

From a neural network learning point of view, a network that forms a memory of its own attractors is a peculiar idea (indeed, the converse is more familiar [27]). Forming an associative memory means that a system forms attractors that represent particular patterns or state configurations. For a network to form an associative memory of its own attractors therefore seems redundant; it will be forming attractors that represent attractors that it already has. However, in forming an associative memory of its own attractors the system will nonetheless alter its attractors; it does not alter their positions in state configuration space, but it does alter the size of their basins of attraction (i.e. the set of initial conditions that lead to a given attractor state via local energy minimisation).

Specifically, the more often a particular state configuration is visited the more its basin of attraction will be enlarged and the more it will be visited in future, and so on. Because every initial condition is in exactly one basin of attraction it must be the case that some attractor basins are enlarged at the expense of others. Accordingly, attractors that have initially small basins of attraction will be visited infrequently, and as the basins of other, more commonly visited attractors increase in size, so these infrequently visited attractors will decrease. Eventually, with continued positive feedback, one attractor will out-compete all others, resulting in there being only one attractor remaining in the system.

But what has this got to do with resolving the constraints that were defined in the original connections of the system? One might expect, given naïve positive feedback principles, that the one remaining attractor would have the mean or perhaps modal global utility of the attractor states in the original system; but this is not the case (Fig.1.d). In order to understand whether the competition between attractors in a selfmodelling system enlarges attractors with especially high total utility or not, we need to understand the relationship between attractor basin size and the total utility of their attractor states. At first glance it might appear that there is no special reason why the largest attractor should be the 'best' (highest utility) 
attractor - after all, it is not generally true in optimisation problems that the basin of attraction for a locally optimal solution is proportional to its quality. But in fact, existing theory tells us that this is indeed the case [26] for systems that are additively composed of many low-order interactions. Specifically, in systems that are built from the superposition of many symmetric pair-wise interactions, the height (with respect to total utility) of an attractor basin is positively related to its width (the size of the basin of attraction), and the globally optimal attractor state has the largest basin of attraction [15]. One must not conflate, however, the idea that the global optimum has the largest basin, with the idea that it is a significant proportion of the total configuration space and therefore easy to find: In particular, the global optimum may be unique, whereas there will generally be many more attractors that lead to inferior solutions, and importantly, the basins of these sub-optimal attractors will collectively occupy much more of the configuration space than the basin of the global optimum.

Given that high utility attractors have larger basins than low utility attractors, they are therefore visited more frequently and therefore out-compete low utility attractors in this self-modelling system. Thus, (in the limit of low learning rates such that the system can visit a sufficient sample of attractors) we expect that when a dynamical system forms an associative memory model of its own utility maximisation behaviour it will produce a 'model' with ultimately only one attractor, and this attractor will correspond to the globally optimal minimisation of constraints between variables in the original system [15].

This is not an entirely satisfactory conclusion however. It implies that the system only fixes on the global optimum because the global optimum has already been visited many times in the past. But this is not the full story. A final part of the puzzle is provided by the well-known ability of Hebbian learning to generalise training patterns and create learned attractors that represent new combinations of common features from the training patterns rather than the training patterns per se. In associative memory research the creation of such 'spurious attractors' is generally considered to be a nuisance [28], but it in fact represents a simple form of generalisation that is important for our results [1]. Producing new attractor states that are new 
combinations of features (sub-patterns) observed in the training patterns [29] enables the globally optimal attractor to be enlarged even though it has not yet been visited. Basically, this occurs because when Hebbian learning is applied to a training pattern it not only has the effect of enlarging the basin of attraction for this pattern, but also it enlarges the basin of attraction for all configurations in proportion to how many behaviour-pairs they share in common. The global optimum is, by definition, the configuration that has the most simultaneously satisfied constraints, and this ensures that, on average at least, it tends to share many behaviour combinations in common with locally optimal configurations (each of which has many constraints simultaneously satisfied but not as many as globally possible).

In addition, it is important to recognise how the separation of the timescales for behaviours on the network and behaviours of the network (i.e. changes to network structure) influence this result. Getting the timescale of the changes to network structure correct is equivalent to the problem of setting the learning rate correctly in a neural network. If connections are modified too slowly then learning is unnecessarily slow. And if learning happens too quickly the network will only learn the first local optimum it arrives at, or worse, if the learning rate is really high, the system could get stuck on some transient configuration that is not even locally optimal [15]. More generally, if most learning happens at or near random initial conditions then the patterns learnt will be similarly random. It is therefore essential that the system is allowed to relax to local optima, and that most learning therefore happens at local optima, so that the patterns learned are better than random. But if the system is not perturbed frequently enough or vigorously enough, and consequently spends all of its time at one or a few local optima, the system will simply learn these attractor configurations and will not generalise correctly.

Lastly on this equivalence, it should be noted that the Hopfield model is not new [13], and its capabilities for Hebbian learning are well known [27]. However, here we provide a reinterpretation of the system, staging it in a generic, game-theoretic network scenario, which opens up the possibility of reinterpretation of some of the analytically solved variants of the Hopfield model (e.g. [30,31]). 


\subsection{Why Adopt a Habitual Policy?}

In the model presented here we mandate that agents follow a habitual policy. Habituation, or a preference for the status quo, is not an uncommon phenomenon in natural systems, but if such a policy was optional, would it be in the self-interest of agents to adopt it? Related work examines this question in detail and shows that when agents can directly alter their utility by altering their constraints with other agents, selfinterested agents will always alter those constraints in a Hebbian manner, thereby causing habituation [1]. However, in the current model, we are examining a subtly different scenario where agents can alter their perception of an interaction but cannot alter the true value of that interaction. We have shown successfully in this paper that a (mandated) policy of altering perceptions in a habituating manner has the effect of increasing the future likelihood of the system reaching attractors that have high true utility. But since altering a perception per se has no direct effect on true utility a selfish agent has no direct or immediate reason to alter its perceptions unless a) that change in perception causes the agent to immediately alter its behaviour, b) this change in behaviour immediately increases utility. So, since altering perceptions in a habituating manner necessarily has the effect of reinforcing or stabilising the current behaviour, selfish changes to perceptions will only be habituating when reinforcing the current behavioural state is desirable.

In general, it is not guaranteed that the current behavioural state, nor therefore reinforcement of the current behavioural state, is desirable for an agent. But, assuming that an agent updates its behaviours rapidly compared to its perceptions (see the separation of timescales assumption [15]), as is the case here, the agent will most of the time exhibit the behavioural choice that is currently the best compromise of its conflicting interests. Whenever this is the case, any change to perceived utility that increases the magnitude of the current behaviour will be preferred over a change to perceived utility that decreases the current behaviour. Thus although it is not the case for all behavioural configurations that the changes to perceptions that increase utility are Hebbian, they will be Hebbian for the behavioural configurations that are most common under the separation of timescales condition. In short, under these conditions, Hebbian 
learning (i.e. preferring the current state) becomes equivalent to reinforcement learning (i.e. preferring what's good). This reasoning suggests that, given the option, selfish agents would change preferences in the same manner as the habituation policy we mandate in this paper - not because of the future increases in total utility they afford, but because of immediate individual utility gains.

However, one final complication is introduced by the possibility that the current behavioural state may already be locally optimal in magnitude as well as sign (in particular, if behaviours are discrete). In this case, there is no change to behaviour that can increase utility. But even here, note that an anti-Hebbian change to perceptions might cause behaviours to move away from this locally optimal state, causing a decrease in utility, whereas a Hebbian change to perceptions is at worst neutral. This selection against antiHebbian changes (plus stochastic variation) is sufficient under some circumstances for a selfish agent to exhibit a systematic trend towards Hebbian changes [1,32,33].We have been studying these mechanisms in several evolutionary scenarios: co-evolving species in an ecosystem that can alter the coefficients of a Lotka-Volterra system [34,35], co-evolving symbiotic relationships that affect co-dispersal probabilities [33], and the evolution of a gene interactions that affect the correlation of phenotypic traits [32]. This work provides examples of the more general concept we refer to as 'social niche construction' - the ability of an agent to create social contexts that subsequently alter social behaviour $[36,37,38,39,40]$. Collectively these works suggest that reinforcing the status quo, and thereby exhibiting a tendency to recall or recreate behavioural patterns exhibited in the past, may be a widespread property of adaptive multi-agent systems [1]. Formal neural network models (where a Hebbian assumption is normal) then help us to better understand the conditions where this tendency will increase total welfare $[1,15]$.

\subsection{Timescales of Selfishness}

Habituating agents start out behaving exactly the same as default agents - making individual utilitymaximising behavioural decisions. However, as they change their perceived utilities this begins to influence their decisions, causing them to adopt different behaviours than default selfish agents. Thus, even though 
the previous section shows that habituation can be motivated by immediate selfish interests, the habituated policy so created can cause agents to subsequently adopt behaviours that decrease individual utility. Since ultimately these new behaviours result in higher total utility, they are arguably cooperative not selfish. However, since the habituated policy leads to higher global utility in the long term, and global utility is simply the sum of individual utilities, it must mean that under the habituated policy, on average each agent is likely to have higher individual true utility in the long term than under the default selfish policy. So, which agents are the selfish ones - the default agents or the habituated agents?

We propose a novel resolution to this inconsistency: both policies are selfish, but simply selfish over different timescales. That is, a rational selfish agent that was attempting to maximise its utility in the immediate term would adopt the default policy over the habitual policy. But, given a choice and the appropriate information, a rational selfish agent that was attempting to maximise its utility in the long term would adopt the habitual policy over the default policy because, although this policy might cause it to suffer utility decreases in the short term, on average it leads to higher total-utility attractors at equilibrium.

This implies a refined definition of 'selfishness': A behavioural policy can be described as 'selfish' if it maximises individual utility (given the available information) on some timescale or other. Such selfish behavioural policies can be sub-categorised based on the timescale over which they maximise utility: the behavioural policy's timescale of selfishness. This classification leads to the possibility of having two or more behavioural policies that can equally be said to be 'selfish' (i.e. they each maximise utility on some timescale or other), but nonetheless, because they are selfish on different timescales, these policies may cause different behaviours - even when faced with identical environments.

We therefore describe behavioural policies that optimise utility over some timescale other than the immediate term as (to some extent) long-term selfish. They are selfish policies that have non-immediate timescales of selfishness. For a given system, if there exists a higher utility optimum that cannot be reached by following local gradients, then a policy that systematically reaches this non-local utility optimum would 
be described as long-term selfish. In addition, to reach a non-local utility optimum, it must be that such a policy entails occasionally accepting temporary decreases in utility (otherwise it would be forced to follow local gradients, and hence end up at the local utility optimum). Furthermore, the ability to systematically reach a non-local, higher utility optimum logically implies that such a policy must have access to - in some form or other - information about that system beyond that of local utility gradients. This information must be stored somewhere, and it must influence the behaviour of the agents under this policy. Another way of describing such an information store that affects decision making is a memory: Essentially, we claim that such a long-term selfish policy (or a mechanism, like our habituation mechanism, that causes an agent to act in a manner consistent with long-term selfish policy) necessarily requires (overtly or otherwise) a functional memory that contains or acquires information about the long-term utility of behavioural choices in the system.

Under this classification, the habitual agents of the current model act in manner consistent with a longterm selfish behavioural policy, in the sense that it maximises utility, but does so on a timescale longer than the immediate. The information about long-term utility that such a policy requires - the memory - is distributed, and stored in the preference matrices of all of the agents. Habitual agents (and not default agents) always possess the machinery of such a memory, although though at the start of the simulation it contains no information (and hence at this stage it has no effect on behaviour, and so habitual agents act as default agents). It is comprised of the storage medium (preference matrices), a write mechanism (habituation) and a read mechanism (behaviour decisions of habitual agents are based on perceived utility). And it is only once this memory has been populated with information about non-local utility optima (i.e. after repeated perturbations, so that the system has had chance to experience more than one optimum) that it begins to cause behavioural differences from the default policy. Default agents, on the other hand, do not possess such memory machinery: they have no mechanism that can store information about the system, and hence their behavioural choices are always based solely on immediate utility gradients, 
limiting them to simple, immediate term utility optimisation, which is always destined to find only the local utility optimum.

\subsection{Reinforcement Learning and Fictitious Play}

In the previous sections we have discussed two points that indirectly link the habituation policy to mechanisms of reinforcement learning [43]. The first point is that in the system modelled in this paper, due to the separation of timescales (i.e. that agent behaviours change much faster than agent perceptions), the policy of habituation, or 'do more of what you are currently doing' is effectively equivalent to 'do more of what is good'. The result is that agents learn to prefer behaviours that have been successful in the past which is the central mechanism of reinforcement learning. The second point is that making decisions that are influenced to some degree by historic information (such as by preference matrices in the current model), can be viewed in some cases as if agents were 'attempting to' predict future outcomes. In some systems, even if agents are myopic (such as habituated agents, who have no 'intention' of predicting long term outcomes of their actions) the structure of the system itself can be enough to effectively convert this myopic behavioural policy into one that is indistinguishable from a policy where agents overtly reason about the future by generalising from the past (i.e. reinforcement learning agents). The model shown here has the form of such a myopic system that when considered as a whole has the effect of optimising in a manner that appears very similar to reinforcement learning.

In particular, the behaviour of the model can be linked to the fictitious play algorithm [41,42]. Under a policy of pure fictitious play, each agent records past opponent behaviours and then makes the assumption that opponents will adopt a randomised strategy that selects each behaviour with a probability given by the frequency it has been played by that opponent in the past. The agent then best-responds to the joint strategy (i.e. cross-product) of all of its opponents' strategies. Ignoring the distributed nature of our model, the habitual policy appears to lie under the umbrella of fictitious play algorithms as described for extensive form games (i.e. asynchronous behaviour updating) [42]. Even from this perspective, however, habituation 
differs from the standard description of extensive form fictitious play, largely due to the strong discrepancy in the habitual model between the influence of a given opponent's current behaviour and any of their individual historic behaviours: Habituation effectively involves a fixed historic discounting of influence, where opponents' current behaviours always have significantly more influence on decision making than behaviours from any individual past move (although when acting as a whole, recorded historic behaviours can come to overwhelm the influence of opponents' current behaviours). This therefore links habituation to generalised weakened fictitious play [18], a more general class of fictitious play algorithms that can include similar forms of historic discounting.

There are therefore clear parallels between the current model and traditional systems of reinforcement learning, but there are also important differences to be highlighted. First, theoretically, the recognition that this type of individual learning between agents is equivalent to associative learning familiar in neural networks is important for understanding the global adaptation that results. And second, mechanistically, the current model uses the repeated relaxation protocol and the separation of timescales to enable the associative memory thus formed to generalise over many locally optimal equilibria. Without these dynamical conditions, the behaviour of habituating agents would not be equivalent to reinforcement learning and progressive system-level optimisation would not occur.

\section{Conclusions}

This paper has investigated the effect of a simple distributed strategy for increasing total utility in systems of selfish agents. Specifically, habituating selfish agents develop a preference for coordinating behaviours with those they are coordinating with at the present moment, and henceforth adopt behaviours that maximise the sum of true utility and these preferences. We show that this causes agents to modify the dynamical attractors of the system as a whole in a manner that enlarges the basins of attraction for system configurations with high total utility. This means that after habituation, agents sometimes make decisions about their behaviour that may (at least temporarily) decrease their personal utility but that in the long run 
increases (the probability of arriving at configurations that maximise) global utility. We show that the habituating agents effectively restructure the connections in the network in a Hebbian manner and thus through the simple distributed actions of each individual agent, the network as a whole behaves in a manner that is functionally equivalent to a simple form of learning neural network. This network improves global adaptation by forming an associative memory of locally optimal configurations that, via the inherent generalisation properties of associative memory, enlarges the basin of attraction of the global optima. This work thereby helps us to understand self-organisation in networks of selfish agents and very simple processes that subtly deviate selfish agents in the direction that maximises global utility without overtly prescribing cooperation or using any form of centralised control. 
Acknowledgments: We thank Archie Chapman, Alex Penn, Simon Powers, Seth Bullock and Anna Jewitt for useful discussions.

\section{References}

[1] Watson, R. A., Mills, R., \& Buckley, C. L. (2010). Global adaptation in networks of selfish components: Emergent associative memory at the system scale. Artificial Life 17(3).

[2] Heylighen, F., Gershenson, C., Staab, S., Flake, G. W., Pennock, D. M., Fain, D. C., De Roure, D., Aberer, K., Shen, W. M., Dousse, O., \& Thiran, P. (2005). Neurons, viscose fluids, freshwater polyp hydra-and self-organizing information systems. Intelligent Systems, 18(4), 72-86.

[3] Nettleton, R. W., \& Schloemer, G. R. (1997). Self-organizing channel assignment for wireless systems. Communications Magazine 35(8), 46-51.

[4] Maynard Smith, J. \& Szathmáry, E. (1997). The major transitions in evolution. Oxford: Oxford Univ. Pr.

[5] Dawkins, R., (2006). The selfish gene (3 ${ }^{\text {rd }}$ ed.). Oxford: Oxford Univ. Pr.

[6] Michod, R.E., (2000). Darwinian dynamics: evolutionary transitions in fitness and individuality. Princeton: Princeton Univ. Pr.

[7] Newman, M. E., Barabasi, A. L., \& Watts, D. J. (2006). The structure and dynamics of networks. Princeton: Princeton Univ. Pr. 
[8] Pacheco, J. M., Lenaerts, T., \& Santos, F. C. (2007). Evolution of cooperation in a population of selfish adaptive agents. In F. Almeida e Costa (Ed.), Proceedings of the 9th European conference on advances in artificial life (pp. 535-544). Springer.

[9] Santos, F. C., Pacheco, J. M., \& Lenaerts, T. (2006). Cooperation prevails when individuals adjust their social ties. PLoS Computational Biology, 2(10), e140.

[10] Nowak, M. A. (2006). Five rules for the evolution of cooperation. Science, 314(5805), 15601563.

[11] Taylor, C. \& Nowak, M. A. (2007). Transforming the dilemma. Evolution, 61(10), 2281-2292.

[12] Ohtsuki, H. \& Nowak, M. A. (2006). The replicator equation on graphs. Journal of Theoretical Biology, 243(1), 86-97.

[13] Hopfield, J. J. (1982). Neural networks and physical systems with emergent collective computational abilities. Proceedings of the National Academy of Sciences of the United States of America, 79(8) 2554-2558.

[14] Crosby, D., Stills, S., \& Nash G. (1971). Love the one you're with. New York: Atlantic.

[15] Watson, R. A., Buckley, C. L., \& Mills, R. (2010). Optimisation in 'self-modelling' complex adaptive systems. Complexity (in press)...

[16] Hebb, D. O. (1949). The organization of behavior: A neuropsychological theory. New York: Wiley.

[17] Hinton, G. E., \& Sejnowski, T. J. (1983). Analyzing cooperative computation. In Proceedings of the fifth annual conference of the cognitive science society. New York: Erlbaum. 
[18] Leslie, D. S., \& Collins, E. J. (2006). Generalised weakened fictitious play. Games and Economic Behavior, 56(2), 285-298.

[19] Monderer, D., \& Shapley, L. S. (1996). Potential games. Games and Economic Behavior 14(1), 124-143.

[20] Chapman, A. C., Rogers, A., Jennings, N. J., \& Leslie, D. S. (2010). A unifying framework for iterative approximate best response algorithms for distributed constraint optimisation problems. The Knowledge Engineering Review (in press).

[21] Watson, R. A., Buckley, C. L., \& Mills, R. (2009). The effect of hebbian learning on optimisation in hopfield networks (technical report). Southampton: University of Southampton.

[22] Gross, T., \& Blasius, B. (2008). Adaptive coevolutionary networks: a review. Journal of the Royal Society Interface, 5(20), 259-271.

[23] Van Segbroeck, S., Santos, F. C., Pacheco, J. M., \& Lenaerts, T. (2010). Coevolution of cooperation, response to adverse social ties and network structure. Games 1(3), 317-337.

[24] Gross, T., \& Sayama, H. (2009). Adaptive Networks. Cambridge, MA: NECSI

[25] Wolpert, D. H., \& Macready, W. G. (1997). No free lunch theorems for search. IEEE Transactions on Evolutionary Computation, 1(1) 67-82.

[26] Kryzhanovsky, B. V., Magomedov, B. M., Mikaelian, A. L., \& Fonarev, A. B. (2006). Binary optimization: A relation between the depth of a local minimum and the probability of its detection. Optical Memory and Neural Networks, 15(3), 170-182.

[27] Hopfield, J. J., Feinstein, D. I., \& Palmer, R. G. (1983). 'Unlearning' has a stabilizing effect in 
collective memories. Nature 304, 158-159.

[28] Gascuel, J. D., Moobed, B., \& Weinfeld, M. (1994). An internal mechanism for detecting parasite attractors in a hopfield network. Neural Computation, 6(5), 902-915.

[29] Jang, J. S., Kim, M. W., \& Lee, Y. (1992). A conceptual interpretation of spurious memories in the hopfield-type neural network. Neural Networks, 1992. IJCNN., International Joint Conference on. 1, 21-26.

[30] Coolen, A.C.C., \& Sherrington, D. (1993). Dynamics of fully connected attractor neural networks near saturation. Physical Review Letters, 71(23), 3886-3889.

[31] Kryzhanovsky, B. V., \& Simkina, D. I., \& Kryzhanovsky, V. M. (2009). A vector model of associative memory with clipped synapses. Pattern Recognition and Image Analysis, 19(2), $289-295$.

[32] Watson, R. A., Buckley, C. L., Mills, R., \& Davies, A. P. (2010). Associative memory in gene regulation networks. In H. Fellerman et al. (Eds.), Proceedings of the 12th international conference on artificial life, (pp. 194-203). Cambridge: MIT Press.

[33] Watson, R. A., Palmius, N., Mills, R., Powers, S., \& Penn, A. (2009). Can selfish symbioses effect higher-level selection? In G. Kampis et al (Eds.) Proceedings of 10th European conference on artificial life (ECAL 2009), LNCS 5778 Part II (pp. 27-36). Springer

[34] Lewis, M. (2009). An investigation into the evolution of relationships between species in an ecosystem. Unpublished MSc dissertation, University of Southampton.

[35] Poderoso, F. C., \& Fontanari, J. F., (2007). Model ecosystem with variable interspecies 
interactions. Journal of Physics A: Mathematical and Theoretical, 40(30) 8723.

[36] Odling-Smee, F. J., Laland, K. N., \& Feldman, M. W. (2003). Niche construction: The neglected process in evolution. Princeton: Princeton Univ Pr.

[37] Penn, A. (2006). Ecosystem selection: Simulation, experiment and theory. Unpublished doctoral dissertation, University of Sussex.

[38] Powers, S., Mills, R., Penn, A., \& Watson, R. A. (2009). Social niche construction provides an adaptive explanation for new levels of individuality. In Proceedings of workshop on levels of selection and individuality in evolution (ECAL 2009).

[39] Powers, S., Penn, A., \& Watson, R. (2007). Individual selection for cooperative group formation. In F. Almeida e Costa (Ed.), Proceedings of the 9th European conference on advances in artificial life (pp. 585-594). Springer

[40] Skyrms, B. (2004). The stag hunt and the evolution of social structure. Cambridge: Cambridge Univ. Pr.

[41] Brown, G. W. (1951). Iterative solution of games by fictitious play. In Activity analysis of production and allocation (pp. 374-376). New York: Wiley.

[42] Robinson, J. (1951). An iterative method of solving a game. Annals of Mathematics, 54(2) 296301.

[43] Sutton, R. S., \& Barto, A. G. (1998). Reinforcement learning. Cambridge, MA: MIT Press. 


\begin{tabular}{|c|c|c|c|}
\cline { 3 - 4 } \multicolumn{2}{c|}{} & \multicolumn{2}{c|}{ Player 2 } \\
\cline { 3 - 4 } \multicolumn{2}{c|}{} & A & B \\
\hline \multirow{2}{*}{ Player } & A & $\alpha, \alpha$ & $\beta, \beta$ \\
\cline { 3 - 4 } & & & \\
\hline & & $\beta, \beta$ & $\alpha, \alpha$ \\
\hline
\end{tabular}

Table 1: Payoff for (player 1, player 2). 


\begin{tabular}{|c|c|c|c|}
\cline { 3 - 4 } \multicolumn{2}{c|}{} & \multicolumn{2}{c|}{ Player 2 } \\
\cline { 3 - 4 } \multicolumn{2}{c|}{} & A & B \\
\hline \multirow{2}{*}{ Player } & A & $r$ & $-r$ \\
\cline { 3 - 4 } & & & \\
\hline & & $-r$ & $r$ \\
\hline
\end{tabular}

Table 2: adjustment matrix $A(r=0.005)$ 

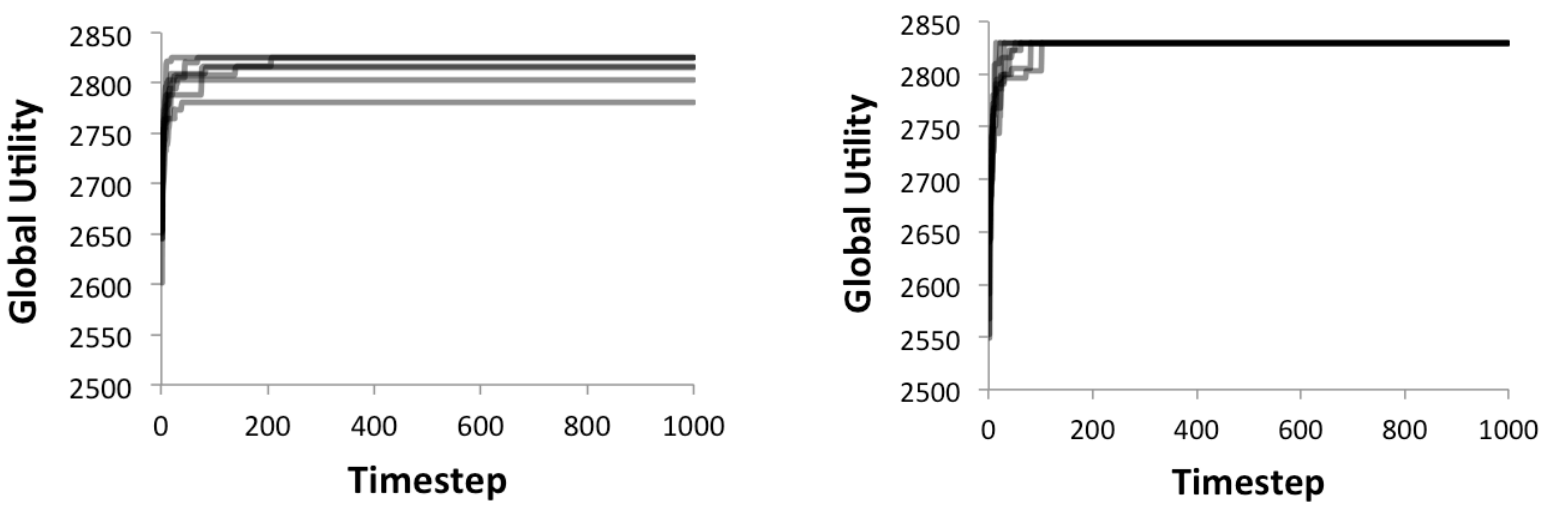

a)

c)
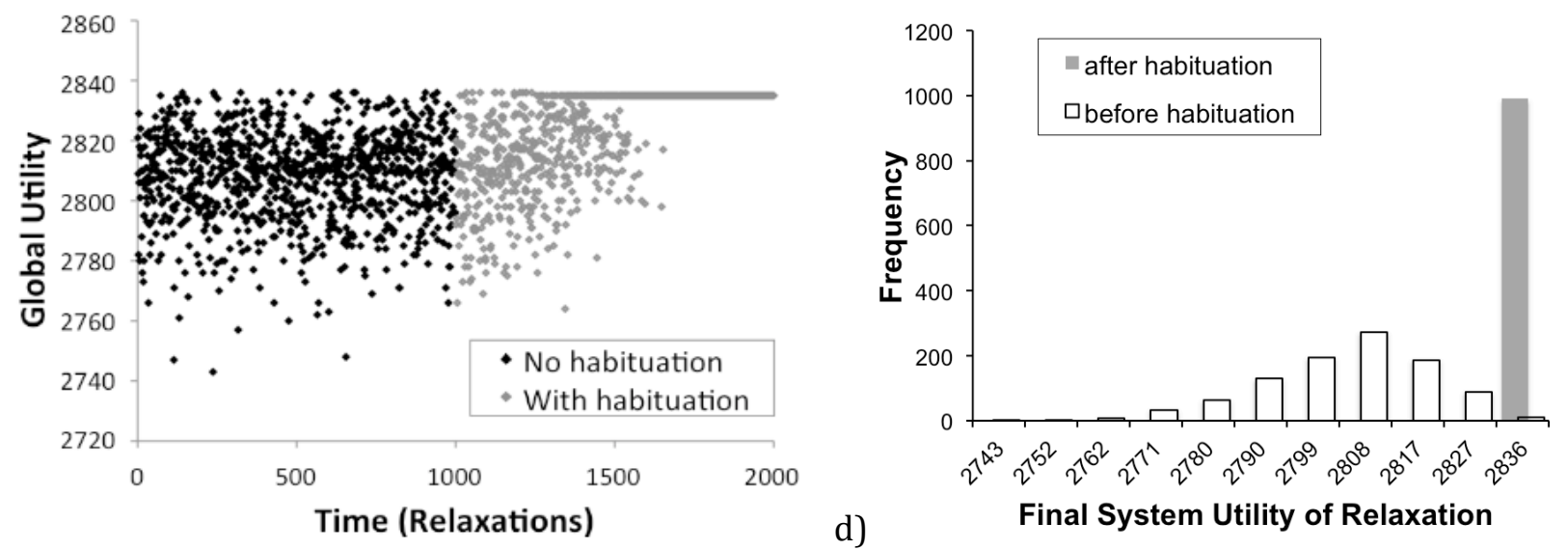

d)

Final System Utility of Relaxation 
Fig.1. Behaviour of the system using default (no habituation) and habituating agents.

a) Some example trajectories of system behaviour before habituation - each curve represents one relaxation $(N=100$, relaxation length $10 N)$ - vertical axis is the total system utility ( $G$, Eq 2); b) utilities of attractor states visited (i.e. end points of curves like those in (a)) without habituation (relaxations 1-1000) and during habituation (relaxations 10012000, $r=0.005)$; c) example trajectories after habituation; d) histogram of attractor utilities before habituation (relaxations 1-1000) and after habituation (relaxations 2001-3000), showing that after habituation the system reliably finds one of the highest total-utility configurations from any initial condition. 